\title{
Nucleon in Nuclear Matter
}

\author{
Aparajita Bhattacharya*, A. Sagari, B. Chakrabarti, A. Chandra \\ Department of Physics, Jadavpur University, Calcutta, India \\ E-mail: pampa@phys.jdvu.ac.in \\ Received August 10, 2010; revised September 14, 2010; accepted September 17, 2010
}

\begin{abstract}
The modification of the properties of nucleon in nuclear medium has been investigated in the context of flux tube model. A nucleon has been described as diquark-quark system connected by flux tube and quasi particle diquark model has been used to describe the diquak constituting the nucleon. The modification of incompressiblity, the Roper resonance etc in the nuclear medium have been investigated. The results are compared with recent experimental and theoretical predictions. Some interesting observations are made.
\end{abstract}

Keywords: Flux Tube, Diquark, Nuclear Matter

\section{Introduction}

EMC effect [1] hints at the modification of the properties of the nucleon in the nuclear environment particularly swelling of nucleon nucleus. The experiment shows that the effect is more as the nuclei become larger and denser. The swelling has largely been interpreted as the effect of attraction of the neighbouring quarks to the quarks constituting the nucleon. This in turn predicts that there are fewer high momentum quarks in the nucleon when it is in nuclear matter as swelling means larger wavelength and small momentum. Recently Seely et al. [2] have reported that the modification of the particle properties do not depend on the mass or density of the nucleus as a whole but only on the immediate neighbourhood within nucleus i.e. the EMC effect depends on the local environment. A number of works have been done in the context of different of models with varied dynamical theory to explain the EMC effect as the swelling of nucleon in the nuclear medium [3]. Oka et al. [4] have shown from the general ground that a composite quantum system will swell (shrink) in an external attractive (repulsive) potential and have concluded that the nucleon in a nucleus swells due to the attraction of the nuclear force. Dukelsky et al. [5] have investigated the EMC effect incorporating mean field acting on the valence quark of nucleon embedded in nuclear medium. They have obtained a swelling of 2 percent which is much lower than the other estimates [4,6]. Kim et al. [7] have investigated the modification of nucleon form factor in nucleus. They have estimated anomalous magnetic moment from the modified form factor and found that it contributes more to the transverse structure function than the longitudinal one. $\mathrm{Wu}$ et al. [8] have studied the modification of the nucleon properties in the Quark Meson Coupling mode (QMC) [9] which includes the effect of the motion of the quark inside the nucleus. They have studied the effective mass, radius, correlated charge density distribution of a nucleon in nuclear matter and have observed that the quark structure plays a crucial role in the description of the nuclear matter and finite nuclei. Mathieu et al. [10] have studied the modification of hadron properties in the context of the flux tube model. They have suggested that nuclear binding and EMC effect are the consequences of the partial deconfinement. In the present work we have investigated the modification of the properties of a nucleon in nuclear medium in the context of the flux tube model. A diquark-quark configuration connected by a flux tube has been considered for a nucleon. The quasiparticle diquark model of diquark suggested by us has been used to estimate the diquark mass. The compressibility, Roper resonance, critical density, swelling of the nucleon in the nuclear medium has been investigated. Some interesting observations are made. In the flux tube model a nucleon is described as a quark and a diquark system connected by a flux tube [10]. The Hamiltonian for an isolated nucleon can be written as:

$$
H=\frac{p^{2}}{2 m}+\sigma r-\frac{4 \alpha_{s}}{3}
$$

where ' $m$ ' is the reduced mass of the system, $p$ is the relative momentum, ' $r$ ' is the distance between quark and diquark, $\sigma$ and $\alpha_{s}$ are the string tension and the strong coupling constant respectively. When the nucleon is in 
nuclear matter the Hamiltonian changes due to the effect of the neighbouring nucleons. In the presence of other nucleon the flux tubes are topologically rearranged such a way that the linear potential of the system is minimum. Considering the effect of one perturbing nucleon, the linear potential of the nucleon can be modified to [10]:

$$
\begin{aligned}
& V_{\text {Leff }}(r)=\sigma r-\frac{\sigma \rho}{6} \frac{\pi}{24} \int I \psi(r) I^{2} \\
& \left(t^{2}-4 u^{2}\right)^{2} \theta(t-2 u) d^{3} r^{\prime}
\end{aligned}
$$

where $r$ and $r^{\prime}$ are the variables that represent the length of the flux tube for the two-nucleon clusterings, $t$ and $\mathrm{u}$ are functions of $\mathrm{r}$ and $\mathrm{r}$ as in Mathieu et al [10]. $\psi(r)$ is typical nucleon wave function, $\rho$ is the nuclear matter density, $\sigma$ is string constant. The one gluon exchange term also would be modified partially due to the presence of the other nucleon and may be represented as in [10]:

$$
\begin{gathered}
V_{\text {Ceff }}(r)=-\frac{4 \alpha_{s}}{3}-\frac{4 \alpha_{s} \rho}{18} \\
\int I \psi(r) I^{2}\left(\frac{2 \pi}{3}\right)(1-z)\left(r^{2}-r^{\prime 2}-4 r r^{\prime}\right) d^{3} r^{\prime}
\end{gathered}
$$

where $z=\cos (\boldsymbol{R}, u)$, here $\mathrm{R}$ is the distance between two nucleons [10]. The Hamiltonian can thus be expressed as:

$$
H=\frac{p^{2}}{2 m}+V_{\text {Leff }}(r)+V_{\text {Ceff }}(r)
$$

In general the contribution to the potential runs as:

$$
V=\int V_{e f f}(r) I \psi\left(r^{\prime}\right) I^{2} d^{3} r^{\prime}
$$

In computing the Hamiltonian we have used the quarkdiquark configuration for a nucleon assuming diquark behaving as a quasi particle suggested by us to estimate the diquark mass. With diquark within, baryon can be described as two-body (involving q-qq) system. In the present study we have considered the proton as a $[\mathrm{ud}]_{0}$ (scalar) diquark and a $\mathrm{u}$ quark system and investigated the modification of the properties of the proton in the nuclear environment.

\section{The Quasi Particle Model of Diquark}

Recently we have proposed a quasi-particle picture of the diquark [11] in analogy to the quasi-particle in usual condensed matter physics. It is well known that the quasi-particles are particle like entities arising in some system of interacting particles. They are low-lying excited states possessing the energy very close to the ground state and the properties of the system and the prpperties of the system can be obtained by investigating the properties of the quasi-particles to a considerable extent. Such states are often observed in the usual condensed matter physics. A crystal electron (with mass m) is subjected to two types of forces namely the effect of crystal field grad $\mathrm{V}$ and an external force $\mathbf{F}$ which accelerates the electron. Under the influence of the two forces, an electron in a crystal behaves like a quasi-particle whose effective mass $m^{*}$ reflects the inertia of electrons which are already in a crystal field [12].

$$
\frac{m}{m^{*}}=1-\frac{1}{F} \frac{\partial V}{\partial r}
$$

We assume similar type of picture for the diquarks. Diquarks are supposed to be fundamental entities and we have considered that their masses get modified due to the two types of forces inside the hadron. One is due to the presence of a background quark field inside the hadron resembling one gluon exchange type interaction and for external force harmonic oscillator type of confinement interaction has been considered. In the context of the potential model potential has been represented as:

$$
V_{i j}=-\frac{\alpha}{r}+\left(F_{i}, F_{j}\right)\left(-\frac{1}{2} K r^{2}\right)
$$

where $\alpha$ is the coupling constant, $F_{i} \cdot F_{j}=-\frac{2}{3}$ for qq interaction [13] and $K$ is the strength parameter, so that,

$$
V_{i j}=-\frac{\alpha}{r}+a r^{2}
$$

where $a=K / 3$ The mass of the diquark in the background of the quark field gets modified in the quasi-particle approximation as it happens in the crystal lattice [12].

While retrieving the $\frac{1}{F} \frac{\partial V}{\partial r}$ part we have considered the one-gluon exchange potential for background field and for external force $F$ we have used the confinement force [11]. Thus we come across:

$$
\frac{\left(m_{q i}+m_{q j}\right)}{m_{D}}=1+\frac{\alpha}{2 a r_{D}^{3}}
$$

where $m_{D}, r_{D}$ are diquark mass and radius respectively, $m_{u}=m_{d}=360 \mathrm{MeV} \quad, \alpha=\frac{2}{3}, \alpha_{s}=0.393 \quad(\mathrm{w}$ i t h $\left.\alpha_{s}=0.58\right), \quad K=241.5 \mathrm{MeV} \mathrm{fm}^{-2}$ [13] the diquarks masses have been estimated as $m_{u d}=506 \mathrm{MeV}$. To estimate the energy we need the wave function for the nucleon. We have used the wave function for the baryon as suggested in the context of the Statistical Model suggested by us [14]. In this model, a hadron is assumed to be consists of a virtual qq(bar) in addition to the valence partners which determines the quantum number of the 
colourless hadron. The quarks, real and virtual, are assumed to be of same colour and flavor so that they may be regarded as identical and indistinguishable and are treated on the same footing. The indistinguishability of the valence quark with the virtual partner calls for the existence of quantum mechanical uncertainty in its available phase space. The valence quarks are assumed to be non-interacting with each other and considered to be moving almost independently in conformity with the experimental finding of asymptotic freedom. However it is considered that the valence quarks move in an average smooth background potential due to their interaction with the virtual sea. With the above considerations an expression for the probability density of the baryon in the ground state has been derived as [14],

$$
|\varphi(r)|^{2}=\frac{315}{64 \pi r_{0}^{\frac{9}{2}}}\left(r_{0}-r\right)^{\frac{3}{2}} \theta\left(r_{0}-r\right)
$$

where $r_{0}$ is the radius parameter of the corresponding hadron and $\theta\left(r_{0}-r\right)$ represents the usual step function. In the modified version of the statistical model, the exchange effect which arises from the tendency of quarks of like spin to keep them apart as suggested by Dirac [15] has been incorporated. The wave function is thus obtained as [16]:

$$
|\varphi(r)|^{2}=A\left(r_{0}-r\right)^{\frac{3 \mu L}{2}} \theta\left(r_{0}-r\right)
$$

The exchange effect has been incorporated through a term $\mu_{L}=\left(\alpha_{e x} / a\right) \frac{d p}{d r}$. The normalization constant is obtained as $A=\left[4 \pi r_{0}^{3 \mu L / 2+3} B\left(3,3 \mu_{L} / 2+1\right)\right]^{-1}$ where $\mathrm{B}$ is the beta function. Using these wave functions as input in the expression (2) and (3), and taking $\mu_{L}=3.66$ [17] the Hamiltonians are obtained as:

$\langle H\rangle=\frac{28.079}{r_{0}^{2}}+0.545 \sigma r_{0}-0.055 \sigma r_{0}^{4}-\frac{3 \alpha_{s}}{r_{0}}+0.312 \alpha_{s} \rho r_{0}^{2}$

And $\langle H\rangle=\frac{46.205}{r_{0}^{2}}+0.312 \sigma r_{0}-0.0088 \sigma r_{0}^{4}-\frac{5.66 \alpha_{s}}{r_{0}}+0.112 \alpha_{s} \rho r_{0}^{2}$

Neglecting the Coulomb term and minimizing the Hamiltonian with respect to radius parameter we come across the following expressions corresponding to equations (12) and (13) respectively as:

$$
r_{0}=\left(\frac{\sigma}{206.048}\right)^{-\frac{1}{3}}\left[1 \pm(1-106.51 \rho)^{\frac{1}{2}}\right]^{-\frac{1}{3}}
$$

And

$$
r_{0}=\left(\frac{\sigma}{584.88}\right)^{-\frac{1}{3}}\left[1 \pm(1-130.19 \rho)^{\frac{1}{2}}\right]^{-\frac{1}{3}}
$$

The compressibility of a nucleon can be expressed as:

$$
K=\frac{1}{3} r_{0}^{2}\left(\frac{d^{2} E}{d r^{2}}\right)
$$

The expression for Roper excitation energy runs as:

$$
\Delta E=\left(\frac{K}{m_{q} r_{0}^{2}}\right)^{\frac{1}{2}}
$$

From the expressions (14) and (15) it has been found that $\rho$ reaches a maximum at $\rho=\frac{\sigma}{106.516} \mathrm{GeV}^{3}$ and $\rho=\frac{\sigma}{130.19} \mathrm{GeV}^{3}$ respectively which corresponds to the real root of the radius parameters and can be defined as the de-confining density or the critical density $\rho_{c}$ of the medium at which the nucleon ceases to exist. We have estimated the compressibility, Roper Resonance with the input of the radii has been estimated with string constant $\sigma=0.219 \mathrm{GeV}^{2} \quad$ [18] from Equations (14) and (15) corresponding to $\rho=0$. The results are displayed in Table 1 and Table 2 corresponding to the energy expressions from Equations (12) and (13) respectively.

Table 1. Compressibility, roper resonance binding energy, critical density with $|\varphi(r)|^{2}$ from (10).

\begin{tabular}{ccccc}
\hline$\sigma\left(\mathrm{GeV}^{2}\right)$ & \multicolumn{2}{c}{ Compressibility } & Roper Resonance & $P_{c}$ \\
\hline & $K_{f}$ & $K_{b}$ & B.E.(GeV) & $1.51 \rho_{0}$ \\
\hline
\end{tabular}

Table 2. Compressibility, roper resonance binding energy, critical density with $|\varphi(r)|^{2}$ from (11).

\begin{tabular}{ccccc}
\hline$\sigma\left(\mathrm{GeV}^{2}\right)$ & \multicolumn{2}{c}{ Compressibility } & Roper Resonance & $P_{c}$ \\
$\mathrm{~K}_{\mathrm{f}}$ & $\mathrm{K}_{\mathrm{b}}$ & B.E.(GeV) & $1.24 \rho_{0}$ \\
\hline 0.219 & 0.635 & 0.517 & 0.012 & \\
\hline
\end{tabular}




\section{Discussions}

In the present work we have investigated the properties of the nucleon in the nuclear matter in the context of the flux tube model considering a nucleon consist of a quark and a diquark connected by a flux tube. Quasi-particle diquark model [14] is used for describing the diquark. The compressibility, Roper excitation energy have been estimated. The results obtained are found to be in the reasonable agreement with the existing predictions. The nucleon shows a swelling in the nuclear medium and the percentage increase in radius at the critical density have been estimated to be of 26 percent. In the context of the non-topological soliton model Wen et al. [19] have estimated the increase in nucleon radius by 10 to 16 percent at normal nuclear matter density whereas Noble [20] has observed the increase in charge radius of nucleon to be 30 percent. Mathieu et al. [10] have observed 25 percent increase in radius at deconfinement density in the context of the flux tube model. Dukelsky et al. [5] have obtained the swelling to be of 2 percent much lower than the existing predictions mentioning the limitation of the model they have used. Chang et al. [21] have investigated the modification of nucleon properties in the context of the Global Colour Symmetry Model and have observed that the nuclear density is 8 times the normal nuclear density at which the radius becomes infinite representing a phase transition. Achtzehnter et al. [22] obtained the critical density to be of the order of normal nuclear density in the context of soliton bag model and argued that the model cannot adequately incorporate the repulsive part of the nucleon-nucleon interaction at small relative distance. The critical density obtained in the present work seems to be lower than the other existing estimates. The difference in compressibility $(\Delta \mathrm{K})$ between the free nucleon and nucleon in the medium at normal nuclear density $\left(\rho_{0}=0.17 \mathrm{fm}^{-3}\right.$ ) has been found to be $0.225 \mathrm{GeV}$ and $0.118 \mathrm{GeV}$ for two version of the Statistical Model used. The reduction of compressibility in medium is found to be substantial in the present investigation. The decrease in compressibility of the nucleon the medium shows that the nucleons in nuclear medium are more diffused than free nucleons. The Roper resonance excitation energy is more in free nucleon than when it is in nuclear medium. The difference has been obtained as $0.029 \mathrm{GeV}$ and $0.012 \mathrm{GeV}$ in the present work. Mathieu et al. [10] obtained the value as $0.021 \mathrm{GeV}$ whereas the most accepted value is of the order of $0.040 \mathrm{GeV}$. In the current investigation we have neglected the contribution from Coulomb term. It may be mentioned that the most uncertainty comes from the radius parameter of the nucleon which has a wide range of values in the literature. The string constant $\sigma$ also have important role in determining the in medium properties and precise knowledge of its value is yet to be determined. It has a range of values [18]. It is interesting to observe that in the current work we have come across an expression where the radius parameter is related to the string constant. If we put radius of the proton to be $0.865 \mathrm{fm}$ which is the charge radius of the proton and suggested to be the equal to the radius parameter defining it as a meso object [23], the string constant would have the value $1.26 \mathrm{GeV}^{2}$ which is quite high compared to the common accepted values. Compressibility also has wide range of values and is under discussion in the current literature. It has been suggested that the information on the compressibility of a system can be obtained from the dynamical properties of the size degree of freedom [24].

\section{Conclusions}

In the current work the quark-diquark model (quasiparticle diquark model) of the nucleon has been used to study the in medium modification of the nucleon properties. Diquarks are now being supposed to be the building block of nucleons particularly after the discovery of the exotics particles. A number of models have been suggested for diquarks [25]. We have also suggested Quasi Particle model and investigated the properties of proton in the framework of the model. Results are found to be interesting. The exchange effect of the quark spin [15] seems to reduce values of all the properties yielding comparatively larger value of the radius parameter. In the present investigation we have dealt with the modification of the nucleon properties due to medium effect through the density of the nuclear matter. However it may be pointed out that recently it has been suggested by Seely et al. [2] that nucleon dependence of quark distribution of nucleon depends on the local environment not on A or the density. More experimental and theoretical efforts are needed to reveal the in medium effect on the nucleon which in turn would help us to know exact nature of the underlying interaction.

\section{References}

[1] J. J. Aubert, et al, "Measurement of Nutron and Proton Structure Function," Physics Letters, Vol. 123B, 1983, pp. 231-236.

[2] J. Seely, "New Measurement of European Muon Collaboration Effect in very Light Nuclei," Physical Review Letters, Vol. 103, 2009, pp. 202301-202305.

[3] M. K. Banerjee, "Nucleon in Nuclear Matter," Physical Review C, Vol. 45, 1992, pp. 1359-1373

[4] M. Oka and R. D. Amado, "Nucleon Size and Attractive Nuclear Force," Physical Review C, Vol. 35, 1987, pp. 
1586-1588

[5] D. Dukelsky, "Nuclear Medium Effects on the Size of the Nucleon Valence Quark Distribution," Journal of Physics $G$, Vol. 21, 1995, pp. 317-330.

[6] K. Brauer, A. Faessler and K. Wildermuth, "Quark-Momentum Distribution in Nuclei," Nuclear Physics A, Vol. 437, 1985, pp. 717-728.

[7] K. S. Kim, M. K. Cheoun, I.-T. Cheon and Y. Chung, "Effect of the Form Factor on Exclusive (e, e'p) Reactions," The European Physical Journal A, Vol. 8, 2000, pp. 131-136.

[8] W. Wu and S. Hong, "Nucleon Properties in the Nuclear Medium" Chinese Physics C, Vol. 32, Suppl. II, 2008, pp. 81-84.

[9] H. Muller and B. K. Jennings, "Nuclear Matter Properties of the Modified Quark-Meson Coupling Model," Nuclear Physics A, Vol. 626, 1997, pp. 966-986.

[10] P. Mathieu and P. J. S. Watson, "A Flux Tube Model of Hadrons in Nuclear Matter," Canadian Journal of Physics, Vol. 64, 1986, pp. 1389-1395.

[11] A. Bhattacharya, A. Sagari, B. Chakrabarti and S. Mani, "Magnetic Moments of the Proton and of Octet Baryons in a Quasiparticle Diquark Model," Physical Review C, Vol. 81, 2010, pp. 015202-1-015202-4.

[12] A. Haug, "Theoretical Solid State Physics," Vol. 1, Pergamon Press, Oxford, 1975.

[13] R. K. Bhaduri, L. E. Cohler and Y. Nogami, "QuarkQuark Interaction and the Nonrelativistic Quark Model," Physical Review Letter, Vol. 44, 1980, pp. 1369-1372.

[14] A. Bhattacharya, S. N. Banerjee, B. Chakrabarti and S. Banerjee, "A Study on the Structure of Proton," Nuclear Physics B, Vol. 142, 2005, pp. 13-15.

[15] P. A. M. Dirac, "Note in Exchange Phenomena in the Thomas Atom," Mathematical Proceedings of the Cambridge Philosophical Society, Vol. 2, 1930, pp. 376-385.
[16] S. N. Banerjee, A. Bhattacharya, B. Chakrabarti and S. Banerjee, "The Properties of a Hadron," International Journal of Modern Physics, Vol. A16, 2001, pp. 201-207.

[17] S. N. Banerjee and N. N. Begum and A. K. Sarear, "On the modified version of Statistical Model," Had. Journal, Vol. 11, 1988, pp. 243-250.

[18] G. L. Strobel, "Roper Resonances as Vibrating Flux Tubes between Quarks," International Journal of Theoretical Physics, Vol. 39, 2000, pp. 115-126.

[19] W. Wen and H. Shen, "Modification of Nucleon Properties in Nuclear Matter and Finite Nuclei," Physical Review $C$, Vol. 77, 2008, pp. 065204-065214.

[20] J. V. Noble, "Modification on Nucleon's Properties in Nuclear Matter," Physical Review Letter, Vol. 46, 1981, pp. 412-415.

[21] L. Chang, Y.X. Liu and H.Guo, "Density Dependence of Nucleon Radius and Mass in the Global Color Symmetry Model of QCD with a Sophisticated Effective Gluon Propagator," Nuclear Physics A, Vol. 750, 2005, pp. 324336.

[22] J. Achtzehnter and W. Scheid, L. Wilets, "Nuclear Matter in the Crystal Soliton Bag Model," Physical Review D, Vol. 32, 1985, pp. 2414-2418.

[23] E. F. Hefter, "Application of the Nonlinear Schrodinger Equation with a Logarithmic Inhomogeneous Term to Nuclear Physics," Physical Review A. Vol. 32, 1985, pp. 1201-1204. doi:10.1103/PhysRevA.32.1201

[24] T. Meissner, F. Grummer and K. Goeke, "Roper Resonances and Generator Co-Ordinate Method in the Chiral Soliton Model," Physical Review D, Vol. 39, 1989, pp. 1903-1912.

[25] R. L. Jaffe and A. Jain, "Implications of the Present Bound on the Width of the $\theta+(1540)$, , Physical Review D, Vol. 71, 2006, pp. 034012-034017. 